\title{
The effects of angle-arm length on judgments of angle magnitude and orientation contrast
}

\author{
PETER WENDEROTH and MICHAEL JOHNSON \\ University of Sydney, Sydney, N.S.W., Australia
}

\begin{abstract}
Acute angles frequently are overestimated, an effect that has been attributed to lateral inhibitory mechanisms. It can be derived from such theories that lengthening the arms of an angle should either reduce or not affect its judged subtense. However, in Experiments 1 and 2, it was found that angles with longer arms were judged larger. In Experiment 3, direct measures of anglearm orientation indicated that orientation contrast does not increase with angle-arm length, that it decreases with distance from the angle vertex, and that these effects are not averaged along the entire length of the arm. It follows that inhibitory mechanisms alone cannot explain why longer armed angles appear larger or why angle arms appear straight rather than bowed. It is suggested that a distinction is required between the orientation and the angular domains and that the latter judgments depend, at least partially, on the space or area within an angle. The fact that angles appear unbowed may reflect the fact that perceived orientation differences occur along an angle's arm only when matches are made to small segments of the arm.
\end{abstract}

It is established that the apparent orientation of one stimulus (e.g., a line) is affected by a stimulus in another orientation. This is the phenomenon of tilt contrast. One widely recognized instance of tilt contrast is the perceptual overestimation of acute angles, and, of all the theories that have been devised to account for this effect, the currently most favored are couched in terms of lateral inhibitory processes (Howard, 1982; Robinson, 1972).

The general notion upon which such theories are based is that each of the two stimulus lines individually induces a certain distribution of excitation and inhibition in the visual cortex. When both lines are presented together, these processes interact: the distribution of excitation corresponding to each line is inhibited more on the side nearer the neighboring contour, resulting in a pair of excitatory peaks that are skewed away from each other. On the assumption that some index of these net excitatory distributions (e.g., the mean, median, or mode) signals the properties of the stimulus, it follows that a pair of angle arms will be perceptually displaced from each other.

Even if this kind of explanation is accepted, the question remains as to whether the neural mechanisms concerned are involved in signaling the position of a stimulus or in coding its orientation. For example, Ganz (1966) offered a theory couched in terms of position domain inhibition, whereas Carpenter and Blakemore's (1973) model is based upon orientation domain inhibition.

Position domain inhibition is often accepted as the explanation of the apparent repulsion of two lines in the same

This research was supported by a grant to the first author from the Australian Research Grants Scheme in 1983 and 1984, for which we express our gratitude.

The authors' mailing address is: Psychology Department, University of Sydney, Sydney, N.S.W. 2006, Australia. orientation, but it is not generally favored as the explanation of orientation contrast. As both Robinson (1972) and Howard (1982) point out, position domain inhibition decreases as the intercontour distance increases. It follows that, for an acute-angle stimulus, the angle's arms should mutually repel each other much more near the vertex, where the contours are close, than elsewhere along the angle's arms. As a result, acute angles should appear bowed, but they do not, except under special conditions (Howard, 1982).

Evidence for the existence of orientation-selective neurons in the visual cortex of cat, monkey, and other species (e.g., Hubel \& Wiesel, 1962, 1968) and for their orderly arrangement into hypercolumns processing field position, ocular dominance, and orientation (Hubel \& Wiesel, 1977) has led numerous researchers to postulate a neural basis for orientation domain inhibition in human vision (e.g., Benevento, Creutzfeldt, \& Kuhnt, 1972; Blakemore, Carpenter, \& Georgeson, 1970; Bouma \& Andriessen, 1968, 1970; Carpenter \& Blakemore, 1973; Lennie, 1971). In this case, if the inhibition is postulated to occur between neural elements signaling orientation independent of position, it might seem that tilt contrast could occur without any bowing's being evident near an angle's vertex (Carpenter \& Blakemore, 1973).

However, Howard (1982) states:

It is not clear to me why lateral inhibition of orientation detectors should not also be limited to the vertex of an angle. After all, most simple orientation detectors respond maximally to a line in a given location and lateral inhibition between them falls off as the lines are separated, as Carpenter and Blakemore showed in their own experiment. I suggest that the presence or absence of bowing does not distinguish between inhibition in the position domain and inhibition in the orientation domain (p. 174). 
In defense of Carpenter and Blakemore, it can be noted that they measured tilt contrast with very short lines $\left(1^{\circ}\right)$ and concluded only that orientation domain inhibition would "generalize for the position of lines, at least up to about $40 \mathrm{~min}$ " (Carpenter \& Blakemore, 1973, p. 301).

Yet even acute angles with long arms do not appear bowed, and the above considerations suggest that they should, whether the inhibition generated is postulated to occur in the position or the orientation domain.

Two possible explanations of the apparent straightness of acute-angle arms are as follows. First, perhaps inhibition is greater near the vertex of acute angles, but observers estimate inclination by averaging along the complete line length (Howard, 1982; Wenderoth, Beh, \& White, 1978). Second, it is possible that only the inhibitory effects at the angle vertex are taken into account by the visual system and that the orientations of the remainder of the angle's arms are "reconstituted," as occurs in the domain of lightness in the Craik-Cornsweet-O'Brien illusion (Frisby, 1979). In the latter case, a stimulus that consists of a midgray patch with a thin black-white edge in its center appears to have an all-black left half and an all-white right half: The central edge alone is used to reconstruct the stimulus.

These possibilities initially led us to conduct experiments on angle estimation. If one of the factors accounting for acute-angle overestimation is lateral inhibition, then making the arms of an acute angle longer would be expected to reduce overestimation if inhibitory effects were averaged along the entire arm length, but would be expected to have no effect on overestimation if "fillingin" occurs, as in the Craik-Cornsweet-O'Brien effect. The first experiment, then, was designed to compare the apparent angular subtense of short-armed and longer armed angles that had equal angular subtense.

\section{EXPERIMENT 1}

No errors occur in matching two angles when the angles differ only in that one has its bisector oriented horizontally and the other has its bisector oriented vertically (Lennie, 1971; Wenderoth \& Johnson, 1982; Wenderoth \& White, 1979). In Experiment 1, therefore, we varied the inclination of one angle's arms until that angle appeared to match another angle whose arms were either shorter, equal to, or longer than the variable angle's arms. The equal-arm condition was included as a control condition, because there is one study in which constant errors did occur in that condition (McRae \& Loh, 1981), for reasons that are not clear (Wenderoth \& Johnson, 1982).

\section{Method}

Apparatus. Displays were produced on the flat screen of a Tektronix 608 monitor (P4 phosphor), interfaced with a Hewlett-Packard graphics translator (1350 A) and a PDP 11/20 computer. Subjects viewed the display from a headrest centered $57 \mathrm{~cm}$ from the screen, so that $1 \mathrm{~cm}$ subtended $1^{\circ}$ of visual angle. A pair of microswitches enabled the observers to indicate whether the variable angle appeared too small or too large, relative to the standard angle.

Stimuli. Each display comprised two angles, one with its bisector vertical (the standard) and the other with its bisector horizontal (the variable). Relative to the center of the screen, the standard vertex pointed down and was $20 \mathrm{~mm}$ up and $20 \mathrm{~mm}$ to the right; the variable vertex pointed left and was $10 \mathrm{~mm}$ down and $10 \mathrm{~mm}$ to the left. The standard angle subtended $20^{\circ}$. Either the standard arm length was fixed at $20 \mathrm{~mm}$ and the variable arm length was 10 , $15,20,25$, or $30 \mathrm{~mm}$ or the variable arm length was fixed at $20 \mathrm{~mm}$ and the standard arm length changed in the same manner. Althought this gave 10 possible conditions (5 arm lengths $\times$ variable/standard length fixed), 2 of these 10 conditions were identical, that is, where both angles had $20-\mathrm{mm}$ arms. We ran this condition only once, but substituted an extreme 10th condition in which the standard arm length was $5 \mathrm{~mm}$ and the variable arm length was $30 \mathrm{~mm}$.

The luminance of all lines, through crossed Polaroid filters, was about $3 \mathrm{~cd} / \mathrm{m}^{2}$, and contrast, defined as $\left(\mathrm{L}_{\max }-\mathrm{L}_{\min }\right) /\left(\mathrm{L}_{\max }+\right.$ $\mathrm{L}_{\min }$ ), was close to 1.0 .

Procedure. The point of subjective equality (PSE) of the variable angle was estimated by means of a double, randomly interleaved staircase technique (Cornsweet, 1962). The starting position of the variable angle was chosen randomly but was restricted to being between angle sizes of $10^{\circ}$ and $30^{\circ}$. Step size was $6^{\circ}$ (each arm moved $3^{\circ}$ ) until 3 reversals had occurred in each staircase, after which it was reduced to $2^{\circ}$ until 5 more reversals occurred in each staircase. The PSE was taken as the mean of these last 10 reversals.

On any trial, the angle pair was flashed for $2 \mathrm{sec}$. Subjects could make a response only during a 3-sec interval following stimulus offset. A response in this period automatically flashed the next stimulus. If $3 \mathrm{sec}$ elapsed without any response, the same stimulus was re-presented.

Instructions. The instructions to subjects were considered extremely important. We did not want judgments to be based upon such variables as the distance (length) between the free arm-ends of the angles, because this distance increases with arm length. The instructions stressed that in comparing angles, attention should be focused on the space within the angles close to the vertex and on the shapes of the angle. It was stressed that distances and areas further away from the vertex should be ignored, otherwise mistakes would occur. The subjects were asked to judge whether the variable angle was too large or too small in angular size, in number of degrees. Postexperimental questioning of subjects convinced us that these instructions had been understood.

Subjects. These were 16 subjects, all volunteers from an introductory course in psychology.

\section{Results}

The results of Experiment 1 were unequivocal but surprising: Long-armed angles were always judged larger than short-armed angles. This is shown in Figure 1.

Analysis of variance with planned contrasts (Winer, 1962 , chap. 4) showed that only linear trend was significant for the descending and ascending functions in Figure $1[\mathrm{Fs}(1,135)=77.31$ and 61.58 , respectively, $\mathrm{p}<.0001]$. The means of these two curves were not different $[\mathrm{F}(1,135)=3.52, \mathrm{p}>.05]$, but the overall mean $\left(-0.53^{\circ}\right)$ was significantly different from zero $[F(1,135)=9.87, p<.005]$. This last result suggests that longer arms have a greater effect on the variable than on the standard angle; for this we have no ready explana- 


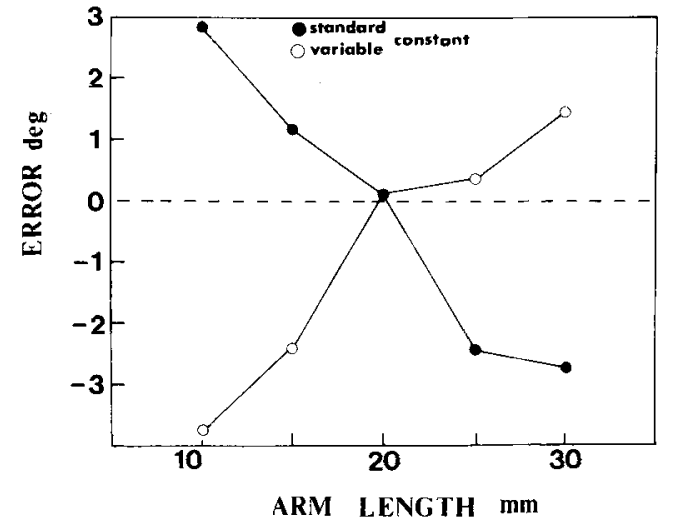

Figure 1. Angle-matching errors obtained in Experiment 1 when either the variable (open circles) or the standard (filled circles) had fixed 20-mm arm length, as a function of the arm length of the other angle. Positive errors indicate that the variable angle was set too large.

tion. However, the result does not imply that constant errors occur when the angles have equal arms because, in that condition, the mean error was $0.11^{\circ}$, with a standard error of $0.64^{\circ}$.

The additional, extreme condition, in which the standard had 5-mm arms and the variable had 30- $\mathrm{mm}$ arms, resulted in a very large error: the variable was set $5.35^{\circ}$ smaller than the standard $\left(\mathrm{SE}=1.02^{\circ}\right)$, and illusion of approximately $25 \%$.

\section{Discussion}

The results of Experiment 1 were unexpected in that we had predicted that longer armed angles would appear either smaller or the same as shorter armed angles, depending upon whether inhibition between the arms was averaged along the arms or whether only inhibition near the vertex was used to signal arm orientation.

Before considering possible reasons for the results, we conducted a second experiment. We were concerned that there might be something special about displays in which the angles were orthogonal, especially ones in which the angle vertices were spatially separated. Hence, the experiment was repeated, this time with the two angle vertices abutting, so that no large eye movements would be required to compare the angles close to their vertices.

\section{EXPERIMENT 2}

Lennie (1971) reported that when two equal angles have a common vertex, but one angle has its bisector horizontal $\left(0^{\circ}\right)$ while the other has its bisector oblique $\left(135^{\circ}\right)$, the oblique angle appears smaller. This illusion is shown in Figure 2, in which both angles are, in fact, $45^{\circ}$.

That the illusion is tied to retinal coordinates can be demonstrated by rotating the page until the originally oblique angle flanks vertical or horizontal. At that point, it is the other (now oblique) angle that appears smaller.

Experiment 2 used displays such as that in Figure 2. We varied the length of the horizontal (variable) angle's arms and the length of the oblique (standard) angle's arms, to see whether the arm-length effect could be used either to counteract (oblique arms longer) or to add to (standard arms longer) the original Lennie illusion.

\section{Method}

Apparatus and Procedures. The apparatus and procedures were identical to those of Experiment 1.

Stimuli. Two angle sizes were used, $20^{\circ}$ and $45^{\circ}$. For each of these two displays, either the standard (oblique) angle's arms were fixed in length at $20 \mathrm{~mm}$ and the variable (horizontal) angle's arms were 10,20 , or $30 \mathrm{~mm}$ long or the variable's arms were fixed at $20 \mathrm{~mm}$ and the standard's arms were 10,20 , or $30 \mathrm{~mm}$ long. The resulting 12 staircase conditions were run in random order.

Subjects. There were 24 subjects, drawn from the same population as Experiment 1.

\section{Results}

The mean errors obtained are shown in Figure 3. The left-hand panel shows the data for the $20^{\circ}$ standard; the right-hand panel shows the data for the $45^{\circ}$ standard. For both angle sizes, when the oblique standard had fixed 20-mm arms (filled circles), increasing the variable's arms

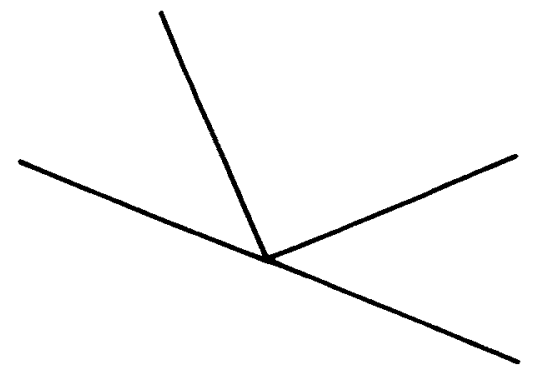

Figure 2. After Lennie (1971): Both angles subtend $45^{\circ}$, but the angle with oblique bisector appears smaller.

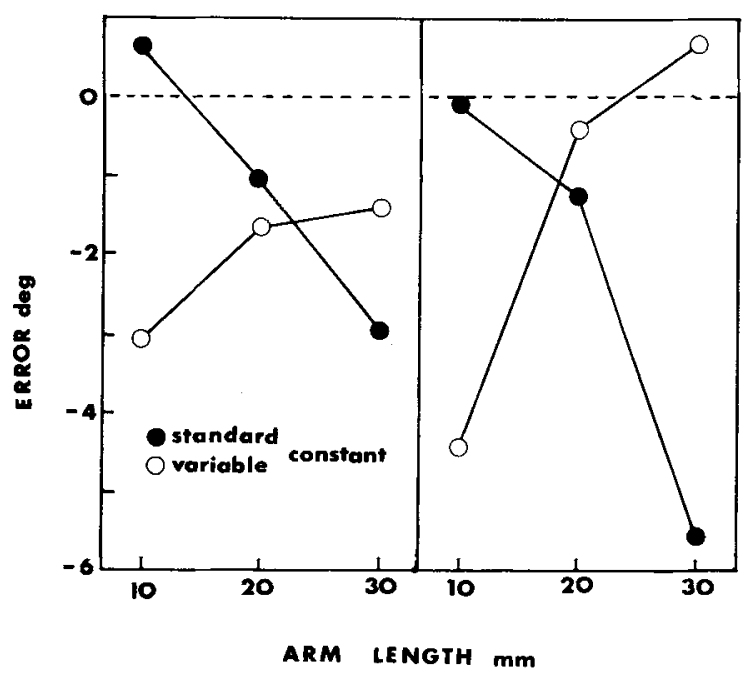

Figure 3. Angle matching errors obtained with Lennie-type displays in Experiment 2. Left panel: $20^{\circ}$ standard. Right panel: $4^{\circ}$ standard. The figure shows matching errors as a function of the arm length of one angle when the other angle, either the standard (open circles) or the variable (filled circles), had a fixed $20-\mathrm{mm}$ arm length. Positive errors indicate that the horizontal variable angle was set too large. 
from 10 to $30 \mathrm{~mm}$ (abscissa) resulted in the variable angle's being set smaller and smaller to match the standard, that is, increasing the length of the variable's arms made it look larger. Conversely, when the horizontal variable had fixed arms (open circles), increasing the length of the standard's arms resulted in the variable angle's being set larger and larger to match it.

These data were analyzed in the same way as those of Experiment 1. For all contrasts, the degrees of freedom were $F(1,253)$.

For the $20^{\circ}$ angles, one function (filled circles) showed a significant linear trend $(F=9.55, \mathrm{p}<.005)$, and the other did not $(F=2.05, p>.05)$. In the case of the $45^{\circ}$ angles, both linear trends were significant $(\mathrm{F}=19.23$ and 22.03, $p<.0005$ in both cases). Quadratic trend was not significant in any case $(F=0.33,0.02,2.20$, and 2.49 , respectively).

When the standard angle was $20^{\circ}$, the overall means of the filled-circle and open-circle functions were not different $(F=1.85, p>.05)$, and this was also true for the $45^{\circ}$ standard $(\mathrm{F}=1.85, \mathrm{p}>.05$ ). Although the mean of the six $45^{\circ}$ conditions $\left(-1.86^{\circ}\right)$ was numerically slightly larger than that of the six $20^{\circ}$ conditions $\left(-1.60^{\circ}\right)$, the difference was not significant $(F=0.29$, $\mathrm{p}>.05)$.

Finally, the grand mean $\left(-1.73^{\circ}\right)$ was significantly different from zero $(F=52.72, p<.0005)$, and this negative mean represents the averaged Lennie illusion over all conditions. The individual Lennie illusions when arm lengths were equal are shown by the four central symbols (i.e., arm length $=20 \mathrm{~mm}$ ) in Figure 3 .

\section{Discussion}

Taken together, Experiments 1 and 2 have shown that acute angles with longer arms are judged larger than otherwise equal angles that have shorter arms. We had predicted that longer armed angles would appear either the same as or smaller than shorter armed angles, depending upon whether the visual system codes orientation solely on the basis of inhibitory interactions near the vertex of an angle, or whether inhibitory effects are averaged over the whole arm length.

Although it seems unlikely that longer armed angles appear larger because inhibition is greater between longer angle arms, it is not certain that perceived arm orientation is determined solely by lateral inhibition, and the possibility remains that longer angle arms appear more displaced from each other than shorter angle arms. Part of the rationale of Experiment 3 was to investigate this possibility.

\section{EXPERIMENT 3}

The design of Experiment 3 was selected not only to find out whether long angle arms appear further apart in orientation, but also to show whether inhibitory effects are or are not averaged along the whole length of an angle arm.
To measure perceived arm orientation and, by inference, the strength of inhibition between angle arms, we used the method of Carpenter and Blakemore (1973). The observer's task (Figure 4) is to set a comparison line (C) parallel to one angle arm (the test arm, $\mathrm{T}$ ), both in the presence and absence of the inducing arm (I). Carpenter and Blakemore (1973) found that small, but systematic, errors occurred when I was absent (pretest) but much larger errors occurred when I was present (test): Line $\mathrm{C}$ was then set much more counterclockwise $\left(C^{\prime}\right.$ in Figure 4), as if to index that $T$ appeared rotated to $T^{\prime}$. The illusion (or inhibitory effect of I) was taken as the difference between the test and pretest setting of line $C$.

In this experiment, we used a shortish line $C(8 \mathrm{~mm})$ and three angle arm lengths $(10,20$, and $30 \mathrm{~mm})$. Line $C$ could occupy one of two positions. One of these was close to the vertex; the other was further from the vertex, although the same orthogonal distance from $T$.

This dual variation of angle-arm length and location of line $\mathrm{C}$ seemed likely to discriminate among the various possibilities discussed above because:

(1) If perceived arm orientation is derived only from inhibition near the vertex, then, no matter where $\mathrm{C}$ is placed along the angle arm, the same effect will be obtained, and this will be independent of arm length.

(2) If perceived arm orientation is derived from inhibition averaged over arm length, the position of $C$ will again be irrelevant but the illusion will decrease as arm length increases.

(3) If inhibition is not averaged along arm length and perceived orientation of a segment of angle arm depends on the inhibition at that point, then, when $\mathrm{C}$ is near the vertex, the effect will always be the same, regardless of
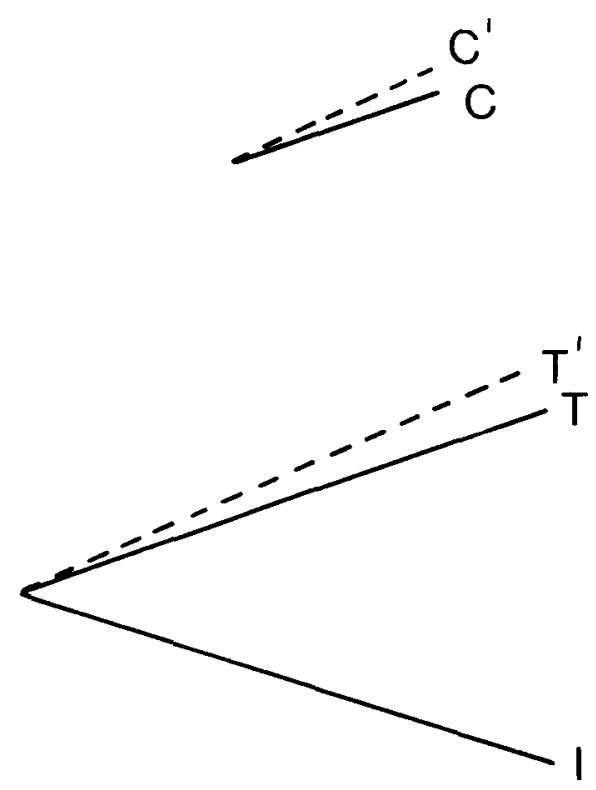

Figure 4. The measurement of orientation contrast. Angle-arm $T$ appears rotated to $T^{\prime}$; hence comparison line $C$ is set to $C^{\prime}$ in order to appear parallel. 
arm length; when $C$ is distal from vertex, similar large effects will occur with short angle arms because the subject is likely to be forced to match to the vertex despite the removal of $\mathrm{C}$; but when $\mathrm{C}$ is distal and angle arms are long, the match will more likely be made to arm segments closer to $\mathrm{C}$ and more removed from the vertex; and so the measured illusion should decrease.

Thus, Experiment 3 aimed to discover, first, whether orientation illusions increase with angle-arm length and, second, to discriminate between the above three possibilities.

\section{Method}

Apparatus. The apparatus was the same as that in Experiments 1 and 2 .

Stimuli. Observers were presented with a $15^{\circ}$ angle, with the inducing arm (I) horizontal and the test arm at $15^{\circ}$ (above I) and with the angle vertex pointing left. The angle size and orientation were chosen to maximize inhibitory effects (Carpenter \& Blakemore, 1973), although a $15^{\circ}$ rather than a $5^{\circ}-10^{\circ}$ angle was selected to ensure clear resolution of the vertex.

The angle's arms were $10 \mathrm{~mm}\left(1^{\circ}\right), 20 \mathrm{~mm}$, or $30 \mathrm{~mm}$ long. The comparison line $(\mathrm{C})$ was $8 \mathrm{~mm}$ long and was always orthogonally separated from arm T by $12 \mathrm{~mm}$. Carpenter and Blakemore (1973) and O'Toole and Wenderoth (1977) have demonstrated that this separation is sufficient to ensure that angle-arm I affects $T$ but not $\mathrm{C}$ (in fact, Carpenter and Blakemore found that interaction between $I$ and $C$ disappeared with a separation of $0.4^{\circ}$, in our case $4 \mathrm{~mm}$ ).

In half of the conditions, line $\mathrm{C}$ had its end nearest the angle vertex $2 \mathrm{~mm}$ from the vertex, measured in the direction of arm T. In the remaining conditions, the vertex end of line $C$ was $12 \mathrm{~mm}$ from the vertex. Line $C$ rotated around its center.

Procedure. Each observer completed the six conditions ( 3 arm lengths $\times 2$ positions of $C$ ) in random order, using staircase methods, as before. However, each of the experimental conditions (line I present) was preceded by a pretest condition in which $\mathrm{C}$ was judged relative to $\mathrm{T}$ with $\mathrm{I}$ absent. This was considered essential because pretest differences could occur merely due to the configurational differences between the stimuli, That is, parallel matches to $T$ might differ when $C$ is nearer the left tip of $T$ than when it is further away. Hence, illusions were taken as the differences between test and pretest parallel matches to $T$.

The starting position of the double randomly interleaved staircases was chosen randomly, with the restriction that the starting orientation of $\mathrm{C}$ was within $\pm 10^{\circ}$ of $\mathrm{T}$. Step size was $3^{\circ}$ until 3 reversals occurred in each staircase and was then reduced to $1^{\circ}$ until 10 more reversals occurred, 5 in each staircase. The PSE was taken as the mean of these last 10 reversals.

Subjects. There were 15 subjects, all volunteers from an introductory course in psychology.

\section{Results}

Figure 5 shows the results of Experiment 3.

Analysis of variance with planned contrasts showed that, when line $\mathrm{C}$ was near the angle vertex (open circles), there was no significant linear or quadratic trend in illusion magnitude as a function of arm length $[\mathrm{Fs}(1,70)$ $=0.15,0.11$, respectively, $\mathrm{p}>.05]$. However, when $\mathrm{C}$ was distant from the vertex (filled circles), illusions decreased as arm length increased, both in linear $(\mathrm{F}=$ $27.46, \mathrm{p}<.0001)$ and quadratic fashion $(\mathrm{F}=12.17$, $\mathrm{p}<.0001)$. The overall means of the two functions were not different $(F=3.97, \mathrm{p}>.05)$.

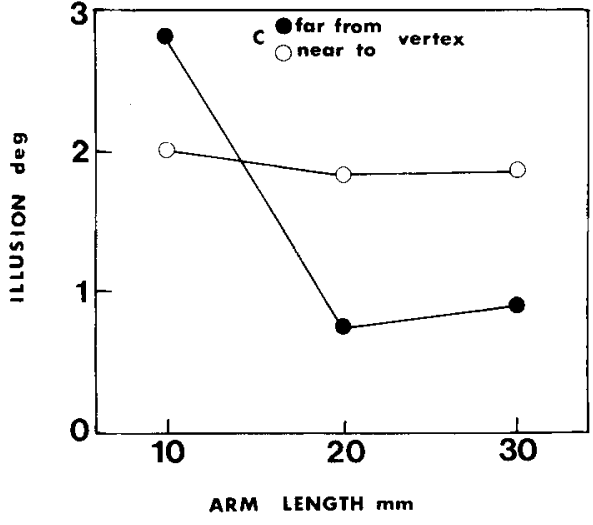

Figure 5. Tilt illusions obtained in Experiment 3 as a function of a $15^{\circ}$ angle's arm length. Comparison line $C$ located near vertex (open circles) or more distantly (filled circles).

This last result suggests that the smaller illusions for 20- and 30-mm arm lengths when $\mathrm{C}$ was distant from the vertex were offset by the larger effect that occurred in the $10-\mathrm{mm}$ condition. That is, when arm length was $10 \mathrm{~mm}$, the illusion in Figure 5 appears larger in the distant- $\mathrm{C}$ than in the close- $\mathrm{C}$ condition. However, inspection of the pretest means indicated that there were configurational artifacts, despite our efforts to avoid them (see Procedure, above).

We had thought that any systematic differences in parallel matching errors that occurred as a function of the position of line $\mathrm{C}$ relative to the angle vertex would occur both in the pretest and the test and, hence, would be factored out in test-minus-pretest difference measures. Recently, Hotopf, Hibberd, and Brown (1983) argued that perspective features exist in a tilted parallel-line display (i.e., lines $\mathrm{C}$ and $\mathrm{T}$ alone, with $\mathrm{I}$ absent) and that these perspective features are destroyed when line $I$ is added. As a result, the pretest lines induce a perspective illusion-they appear to diverge at their upper ends when truly parallel-but this additional component is not present in the test figure. The result is that the parallel matching of $\mathrm{C}$ to $\mathrm{T}$ alone is an inappropriate baseline measure for the tilt illusion because it leads to a spurious overestimation of the effect: $C$ is set an extra amount clockwise in the pretest to offset the apparent divergence of $\mathrm{C}$ and $\mathrm{T}$. There is abundant evidence for the apparent divergence of tilted parallels (Carpenter \& Blakemore, 1973; Wenderoth \& White, 1979), and our data further suggest that as line $\mathrm{C}$ moves higher in the visual field, this apparent divergence increases. Thus, the mean of our three pretest matches when $C$ was near the vertex was $-0.06^{\circ}$; but when $\mathrm{C}$ was distant from the vertex, the mean pretest match was $-0.61^{\circ}$. Consequently, if Hotopf et al. (1983) are correct, all of the C-distant illusions are inflated because in the absence of line I, but not in its presence, parallel lines appear to diverge more at their upper ends when $C$ is higher in the visual field. This artifact could explain the apparently larger illusion in the $10-\mathrm{mm}$ armlength condition when $C$ was distant from the vertex. We 
have pursued this issue elsewhere (Wenderoth \& Johnson, in press).

\section{GENERAL DISCUSSION}

The results of this experiment indicate, first, that longer acute-angle arms do not appear more displaced from each other in orientation than do shorter angle arms. To the extent that parallel matches index inhibition, inhibition does not increase with arm length.

The data are not consistent with two of the three possibilities discussed earlier. First, if arm orientation were derived solely from inhibition near the angle vertex, the two functions in Figure 5 would be superimposed and horizontal. Second, if inhibitory effects were averaged along the length of an angle arm, the two functions would again be superimposed but decreasing. Figure 3 is consistent only with the hypothesis that the perceived orientation of a segment of an angle's arm depends on the inhibition at that point, with inhibition decreasing as separation between the angle's arms increases. It is clear also that the judged size of acute angles depends on factors other than inhibitory effects, since, in Experiments 1 and 2, longer armed angles were judged larger.

One important conclusion, then, which seems to follow from these results, is that it is essential to distinguish between judgments in the orientation domain and judgments in the angular domain. Once before, we reported angular judgments that seemed not to be explicable solely in terms of the perceived orientation of angle arms (Wenderoth \& White, 1979). More recently, Kawabata (1981) has added to this evidence. He noted that when Carpenter and Blakemore (1973) added a third line to the angle display in Figure 4, a line that began at the angle vertex but made a small angle with line $T$, errors in matching $C$ to $\mathrm{T}$ decreased. This effect was attributed to a disinhibitory effect of the third line on line I. Kawabata, however, used logic similar to ours and had subjects match a variable angle to such a three-pronged angle. He found that the three-pronged angle appeared larger and concluded that "angle perception can not be obtained from a difference between the perceived orientations of the angle arms" (Kawabata, 1981, p. 703).

If acute-angle overestimation depends upon factors other than inhibitory effects and perceived arm orientation, then what might those factors be? One possibility is that, despite our careful instructions to subjects, they were influenced by the fact that as arm length increases, the total space within the angle increases. After all, the space enclosed by an angle of fixed arm length does increase with angle size, so that it may be unavoidable to take this space into account in judging angle magnitude. Kawabata's results might then reflect the fact that filled space generally is overestimated relative to unfilled space (Robinson, 1972).

The fact that angle size and angle-arm orientation are correlated perfectly in Euclidean space but not in judgmental space does not imply necessarily that perceptual space is non-Euclidean. Rather, it implies merely that different judgmental tasks probably rest on different variables. Perhaps similar logic applies to the domains of orientation and straightness. For example, Levinson and Frome (1979) showed that inspection of a grating can make a higher frequency grating look even higher while simultaneously causing each individual bar to appear thicker. Their explanation of this apparent paradox was that observers "discount all parts of the visual field except those relevant to their task" (p. 1426). The same idea would explain why angle arms do not appear bowed: Parallel matching to isolated segments of an angle arm reveal orientation differences along the angle arm, but such differences are not evident in judgments of the straightness of the complete arm. An interesting experiment would be gradually to increase the length of the comparison line, C: presumably this would force the observer to average orientation along the angle arm.

\section{REFERENCES}

Benevento, L. A., Creutzfeldt, O. D., \& Kuhnt, U. (1972). Significance of intracortical inhibition in the visual cortex. Nature, New Biology, 238, 124-126.

Blakemore, C., Carpenter, R. H. S., \& Georgeson, M. A. (1970). Lateral inhibition between orientation detectors in the human visual system. Nature, 228, 37-39.

Bouma, H., \& ANDRIESSEN, J. J. (1968). Perceived orientation of isolated line segments. Vision Research, 8, 493-507.

Bouma, H., \& ANDRIESSEN, J. J. (1970). Induced changes in the perceived orientation of line segments. Vision Research, 10, 333-349.

CARPENTER, R. H. S., \& Blakemore, C. (1973). Interactions between orientation in human vision. Experimental Brain Research, 18, 287-303.

CoRnsweEt, T. N. (1962). The staircase method in psychophysics. American Journal of Psychology, 75, 485-491.

FrisBY, J. P. (1979). Seeing: Illusion, brain and mind. Oxford: Oxford University Press.

Ganz, L. (1966). Mechanisms of the figural aftereffects. Psychological Review, 73, 128-150.

Hotopf, W. H. N., Hibberd, M. C., \& Brown, S. A. (1983). Position in the visual field and spatial expansion. Perception, 12, 469-476.

HowARD, I. P. (1982). Visual spatial orientation. New York: Wiley.

Hubel, D. H., \& Wiesel, T. N. (1962). Receptive fields, binocular interaction and functional architecture in the cat's visual cortex. Journal of Physiology (London), 160, 106-154.

HubEL, D. H., \& WiEsEL; T. N. (1968). Receptive fields and func tional architecture of monkey striate cortex. Journal of Physiology (London), 195, 215-243.

Hubel, D. H., \& WIESEL, T. N. (1977). Functional architecture of macaque monkey visual cortex. Proceedings of the Royal Society (London) Series $B, 198,1-59$.

Kawabata, N. (1981). Analysis of the angle perception based on the angle matching technique. Vision Research, 21, 701-703.

LENNIE, P. (1971). Distortions of perceived orientation. Nature, New Biology, 233, 155-156.

Levinson, J. Z., \& Frome, F. S. (1979). Perception of size of one object among many. Science, 206, 1425-1426.

MCRAE, A. W., \& LoH, H. K. (1981). Constant errors occur in matched reproduction of angles even when likely biases are eliminated. Perception \& Psychophysics, 30, 341-346.

O'TOOLE, B. I., \& WENDEROTH, P. (1977). The tilt illusion: Repulsion and attraction effects in the oblique meridian. Vision Research, 17, 367-374.

RoBinson, J. O. (1972). The psychology of visual illusion. London: Hutchinson. 


\section{WENDEROTH AND JOHNSON}

Wenderoth, P., Beh, H., \& White, D. (1978). Perceptual distortion of an oblique line in the presence of an abutting vertical line. Vision Research, 18, 923-930.

Wenderoth, P., \&OHNSON, M. (1982). Constant errors do not occur in the matched reproduction of angles. Perception \& Psychophysics, 32, 388-392.

WENDEROTH, P., \& JoHnson, M. (in press). What is the appropriate control for the tilt illusion? Perception.
Wenderoth, P., \& White, D. (1979). Angle-matching illusions and perceived line orientation. Perception, 8, 565-575.

WINER, B. J. (1962). Statistical principles in experimental design. New York: McGraw-Hill.

(Manuscript received May 24, 1984; accepted for publication September 24, 1984.) 